\title{
Twitterverse: The birth of new words
}

\author{
Olga Klymenko*
}

\begin{abstract}
New words are continuously being coined on Twitter, which is regarded as a means of creating and distributing new words, concepts and ideas. Our research focuses on the way Twitter's apt naming has become one of the richest sources of brand-related word-formation. The current study has identified various productive patterns of new Twitter word formation, such as affixation, compounding, blending, abbreviation and the powerful mechanism of lexical analogy. We have also identified the most common models used in coining new units of each type. The creation of new Twitter words has various functions and effects: establishing a closer social connection; jocular/mocking function; novelty and originality; desire to follow fashion; and denominative/labelling function. We conclude that in the era of an ever-growing amount of data, the popularity of Twitter has become a breeding ground for new brand-related words that have enriched the modern English vocabulary.
\end{abstract}

Keywords. word-formation; affixation; compounding; blending; abbreviation; lexical analogy

1. Introduction. No living language remains stable, its vocabulary is constantly growing and changing. Hundreds of new words are coined every day in social networks like Twitter, which has become a "gold mine for scholars in fields like linguistics, sociology and psychology who are looking for real-time language data to analyze" (Zimmer 2011). The likely reason of significant popularity of Twitter, suggested by Paul McFedries, is the open-endedness of the question "What's happening?", which a user sees updating their status. The author goes on to explain his point: "it opens up a world of new questions: What are you reading? What great idea did you just come up with? What are you worried about? What interesting person did you just see or hear? What great information did you stumble upon on the Web? What hilarious video would you like to share?" (McFedries 2009:13)

The social media Twitter influences the dynamics of language development and change, the patterns of language variation and linguistic standardization, transforms the relationship between standard and colloquial languages. Nowadays new words coined on 'Twitter' refer to spheres other than social networking and information technology as a whole - journalism, entertainment and so on, which means that the phenomenon of Twitter has acquired influence far beyond its initial area of use, as well as that it is actively utilized by people of all professions, not necessarily connected to IT.

Online data is increasingly being used to research English language variation and evolution. One study uses Twitter to map out the way in which new words become popular, and highlights the power of modern tools in analyzing language variation (Grieve, 2016). However, despite 'Twitter'-related publications (McFedries, 2009), most of them regard this social network as a means of distribution of new words, concepts and ideas, rather than creation thereof. This research focuses on the way Twitter's case of apt naming has become one of the richest sources of brand-related word-creation in the recent history.

\footnotetext{
*Author: Olga Klymenko, Zaporizhzhia National University (olga.klymenko.zp@ gmail.com).
} 
Twitter provides an immense resource for new vocabulary items and many innovative terms are based on the words 'twitter', 'tweet' and their clipped forms. People choose to create new 'Twitter'/'tweet'-based units on account of their attractiveness, humorousness or because they allude to their models and therefore are easier to be understood and memorized. Examples of new-coined words include: specialized terms to name appropriate processes and tools, creative vocabulary items coined as cryptic in-group slang, occasionalisms meant to play on words and jocularly attract attention, nonce-words created for stylistic reasons.

In this study "new word" is used as an umbrella term covering both new words accepted by the speech community and new words coined for a particular occasion. I use terms "new word", "new coined word", "new created word" interchangeable as synonyms because all of them "deal with creation of a new word using the available resources of the language of the community; the difference is merely a matter of whether speakers pick up the new word" (Bauer, Lieber, Plag 2013:30). "Twittologism" is a new word or phrase specific to Twitter, created on Twitter, associated with Twitter, coined on the words 'twitter', 'tweet' and their clipped and abbreviated forms. Throughout this study reference to 'Twitter'/ 'tweet'-based words may interchangeably be used alongside reference to 'Twitter' words.

The research questions that are addressed in this work include:

- What is the role of different patterns and models in 'Twitter' word-formation processes?

- How can newly coined 'Twitter' words be associated with their models? Are there any preferable models which are most frequently used?

- What are the functions and effects of new 'Twitter' words?

- What are the factors that favor and motivate new 'Twitter' word-formation and which ones are more important?

2. Data. Since the earliest years of its inception Twitter has led to the emergence of an evergrowing stockpile of new words associated with it: from communication features of it and types of actions you can do with it, to characteristics and descriptions of those using it. The exact number of new words created under its influence is hard to be accurately counted and registered. The database constructed for this study gathers data drawn from various online sources and websites The Twitter Dictionary aka Twittonary (Twitter + Dictionary) and websites like Twittonary.com and Twittords.com list more than a hundred of new 'Twitter'/ 'tweet'-based words. Numerous creations with words 'twitter' or 'tweet' have been coined also in informal communication on social media. Wordspy - The Word Lover's Guide to New Words by Paul McFedries keeps track of emerging vocabulary in the English Language and Aaron Peckham's Urban Dictionary with thousands of new slang words being submitted every day by ordinary people (Twitter-users). Advanced searches in the online dictionaries and on the websites, restricted to the words, which are generally indicated as being created after the words 'twitter' or 'tweet', allow download large amount of corpora-based data, including these words, phrases or collocate frequency. The current study of 'Twitter' corpora (986 items) provides a quantitative analysis of particular patterns and most frequently used word-formation models. As the list of main sources shows the database comprises items attested in corpora and online dictionaries and new creations attested only in the web sources - websites and social media but nevertheless important for the English language evolution and impact of the social media like Twitter on contemporary vocabulary.

The methodology used for data collection combined advanced search with manual selection. Advanced search tools were used for the selection of 'Twitter'-words in online resources. For 
instance, the Urban Dictionary allows for search by entry and also provides cross-references for formally and semantically related words, which may be either the model or the target formation depending on the earliest attestation. The description and contexts provided by compilers helped to make a choice between relevant and irrelevant cases. New 'Twitter'/ 'tweet'-based vocabulary added to the Wordspy website also offers a manual search option, definition and information about the word's etymology and examples of contextual meanings.

3. Analysis. Clarification is to be made concerning the difference among novel words and phrases. Angelo (1991:2) included among the new words in his dictionary not only single words and compounds but also idiomatic phrases. The new words in analyzed dataset include only morphological neologisms (compounds, affixed words, blends, abbreviations, multi-lexical words used as words on Twitter). They are classified according to their morphological category. This morphological categorization is meant to show the frequency and productivity of new 'Twitter'/ 'tweet'-based patterns and models across morphological types of word-formation. The morphological types of new coined words that have been included in dataset are as follows:

\subsection{DERIVATION.}

\subsubsection{SUFFIXATION. The analysis of new 'Twitter'-words enables us to identify:}

- Noun-forming suffixes: -er (twitterer/tweeter "a person who uses Twitter"); -ing (tweeting "communication via Twitter"); -ness (twitterness "contribution of a particular user to Twitter); -ie (tweetie "sweet application for Twitter to use on iPhone or mac"); -ese (twitterese "the language used in posting Twitter messages"); -ia (twitteria "excessive use of the social networking site Twitter.com"); -ation (twitteration "the feeling you get when a friend you've been following on Twitter finally follows you back"); -ism (Twitterism "deep insights about life in 140 chars or less" or tweetism "life affection by Twitter"); -hood (twitterhood "Twitter community"); -dom (twitterdom "the world of Twitter (the microblogging service)".

- Adjective-forming suffixes: -ish (twitterish "very active on Twitter"); -able (twitterable "worthy of being put up on Twitter"); -less (tweetless "refraining from using Twitter").

- Verb-forming suffix: -ize (twitterize "to enable a person/object to access Twitter; to show a person the wonders of Twitter).

- Semi-affixes: -head (tweethead "a person on Twitter who tweets stupid, uninteresting things" or "a person who is addicted to Twitter, and twittering"); -worthy (tweetworthy "worthy of typing into your phone on the subway and posting on Twitter" and Twitterwothy "a random occurrence or event that is so incredible and awesome that it makes a person stop and immediately Twitter about it"); -freak (tweetfreak "one who tweets incessantly, constantly and/or needlessly").How can newly coined 'Twitter' words be associated with their models? Are there any preferable models which are most frequently used?

Frequent splinter - o (<typo) contribute to the 'Twitter'-word-formation via schema in Tweeto "a Twittographical error made while tweeting on a keyboard". The slang back-clipping word typo "typographical error" (OED) has been reanalyzed as a complex model (typo+-o) and this reanalysis has conducted to the target tweet-o. Bauer, Lieber and Plag (2013) include -o with the meaning "language production error" among splinters with moderate productivity. Productivity and regularity in use made the shift from frequent splinter to combining form. 
3.1.2 COMBINING FORMS. They have been used to coin new 'Twitter'-based words, include some elements, currently defined in OED and in Green (2010) as a "suffix":

- Neoclassical combining forms: -ology in Tweetology "the study of the Twitter phenomenon and it's impact on human social interaction"; - phobe in Twitterphobe "a person who is afraid of Twitter"; - phobic in twitterphobic "fearing to follow someone of Twitter that they will not follow back".

- Abbreviated combining forms in the nouns: astro- (<astronomy) in astrotweet "a political message posted to Twitter (or other social network), which is written by skilled writers working for political organizations, and meant to appear as if it was generated as spontaneous political expression by ordinary citizens", geo(<geographical) in geotwitter "the geographical location of the most recent tweets", tele- (<television) in teletwitter "experimental open source client" and in the verbs: de(<delete) in detweet "to delete a Twitter post or 'tweet"; - sphere (<atmosphere) in Twitosphere "the online Twitter community" or a derogatory term for the place where ridiculous, meaningless, and tacky content from social networking sites resides on the Internet .

- Secreted combining forms in the nouns: -aholic (<workaholic) in twittaholic "one who is addicted to Twitter, who cannot live without it"; -erati (<literati) in Twitterari "the Tweet elite, whose feeds attract thousands of followers"; - zine (<magazine) in Twitterzine "a horridly bad concept for a magazine, consisting of entire articles with 140 characters or less written on twitter"; - philia in twitterphilia "updating Twitter at least 5 times an hour, every day" and Tweetophilia "a serious condition in which one suffers from hemorrhaging tweets at a dangerous rate, none of the natural filters or editing agents are present to stop the flow"; - azzi (<paparazzi) in Twitterazzi "the people who having a celebrity or pseudo-celebrity on sight, immediately snap a picture of said famous person and tweets about it"; - tainment (<entertainment) in Twittertainment "entertainment though the public content on Twitter"; -errhea (<diarrhoea) in twitterrhea "symptoms: obsessive twittering in a short time span (often pointless tweets posted just to pass the time), twitching, putting @ signs in front of a person's name before texting or emailing them"; -crat (<bureaucrat) in twittercrat "a politico from either party using Twitter as the sole means of communication"; - preneur (<entrepreneur) in Twitterpreneur "someone creating a Twitter-based startup; -orexic ( $<$ anorexic) in twitterexic "a person refraining from Twitter"; - gasm (<orgasm) in twittergasm "when a female describes how physically attractive a guy, usually a celebrity, is in numerous consecutive tweets"; - itis in twitteritis "a term describing a not-so-rare messaging condition linked to the use of Twitter, a micro-blogging technology; -(e)rian (<vegeterian) in twitterian "a Twitter user with a high number of tweets"; -plomacy (<diplomacy) in Twitterplomacy "ranting on Twitter instead of meeting face to face"; - arama (<panorama) tweetarama "the world wide spectrum of tweeting".

\subsubsection{PREFIXATION. Productive prefixes include:}

- Noun-forming prefixes: cyber- (cybertweet "Twitter-based mutual interation with someone you wouldn't speak to in real life"), sub- (subtweet "an insulting or suggestive Twitter post that indirectly mentions another Twitter user, thus reducing the chance that the other person will see the message"). 
- Verb-forming prefixes: mis- (mistweet "a tweet that you later regret having sent"), re(retweet "copy and post another user's Twitter post").

The research proved that affixation is a productive type of 'Twitter'/ 'tweet' wordformation. The number of suffixes involved is limited to 18 compared to the total number of suffixes of Standard English, which, according to various researchers, ranges from 80 to 100 suffixes (Bauer, 1983). The combining forms amount to 23. The number of prefixes is limited to 4. This confirms the general tendency of Modern English for language economy and minimization of the number of lexical word-formation tools.

3.2. Compounding. We divided new 'Twitter' compound words into the following types:

\subsubsection{SIMPLE-STEM COMPOUNDS:}

- Compound nouns: tweetstorm "a flurry of Twitter posts from a particular person or about a particular topic"; beetweet "a buzzing tweet; a 'hot' tweet"; bulltwitter "a twitter user who's not truthful when tweeting"; twittertale "one who tattles on others via twitter; an informer or talebearer of others exploits by tweeting"; Twitterproxy "a ghost writer for a celebrity on Twitter"; tweetthief "a person who steals jokes and material from people on Twitter and tweets them as if they were their own, with no attribution, re-tweet, or credit given"; twitterace "different genders, ethnicities or people in general who use Twitter"; twitterbeast "a person who constantly trawls Twitter looking for amusing or pornographic tweets to amuse his or her friends"; snaptweet "a tweet that includes a photo"; twitterbreak "a moment during something strenuous or dangerous to tweet about it on Twitter"; twitternest "the group of people on Twitter that are following you"; twitterwall "a selection of tweets shown somewhere in the real world"; tweetmeat "people constantly posting on Twitter"; twitterstar "people who rely on having a large number of followers on Twitter as a form of social validation"; twitterbox "a chatter box that uses twitter as their delivery mechanism"; tweethate "when the tweeting audience dislikes a tweeter"; twitteridiot "a person who uses Twitter to broadcast asinine pronouncements, petty complaints, churlish insults, insane misjudgments, misogynistic rantings, reckless accusations, absurd vulgarities, vindictiveness unlimited, and/or unhinged ravings, seemingly unaware or unconcerned with their veracity, propriety or wisdom" or "a person who apparently does not realize how Twitter works, and that others much smarter than one's self will be seeing and reading posted tweets and passing judgment on the character of the writer".

- Compound adjectives: twitterdeaf "not able to 'hear' friends' tweets because follow many other people"; twitterdrunk "tweeting anything that crosses your mind".

- Compound verbs: saytweet "to create a Twitter badge that shows your updates on a picture"; styletweet "to give fashion/style advice using twitter"; beertweet "to drink beer together and talk about...Twitter (and another stuff)"; twitterbomb "to pollute the stream of a Twitter hashtag by tweeting a meaningless, satirical, or spamlike tweet containing the hashtag"; tweetdrink "to tweet while you are drinking".

\subsubsection{DERIVATIONAL COMPOUNDS:}

- Noun + Noun + -er $=\mathrm{N}$ : tweetsitter "one who watches another's twitter feed and deletes any inappropriate tweets as they appear"; tweet-timer "a person that tweets one tweet, yet tells two seperate people its directed towards them"; Tweet-Watcher "someone who stays logged in to Twitter all day but never tweets and watches to see what everyone else has to say"; Twitterposter "a person who is impersonating another person via 
Twitter"; Twitterwriter "a person who writes regularly on Twitter"; Twitternetter "person who tweets from multiple locations"; reportwitter "reporter style twitterers".

- Noun + Noun + -ing $=\mathrm{N}$ : tweetstacking "the practice of posting multiple tweets in a row on Twitter, usually to express something longer than 140 words"; tweet-dropping "a one-way fake conversation with a celebrity"; tweetcasting "live tweeting an event on the social networking site Twitter"; tweetdecking "stealing tweets and tweeting them yourself to a large amount of people"; Twitter-camping "the act of regularly sending tweets"; twitteracting "interacting on Twitter"; twittergrieving "shameless e-weeping over the untimely death of a celebrity".

- Noun + Noun + -ed = Adj.: Twittergrounded "when a person logged on to the social networking site Twitter uses the post and reply features so much within a short period of time, the service temporarily shuts down their tweeting privileges"; twitterpainted "a sexual and joyous emotional feeling of companionship for another that typically induces frequent distractions from daily life caused by daydreaming, ultimately causing love and an intense desire for a long loving relationship"; twitterwasted "when you waste good news by tweeting it rather than sharing with your friends face to face".

- Adjective + Noun + -ing $=\mathrm{N}$ : drunktwittering "the act of posting on Twitter while intoxicated".

- Multiple-stem compound nouns: tweetfacelinkblogging "the action of posting the same statement to multiple social media tools"; tweethangover "when someone becomes physically or emotionally sick after a long tweetbender".

- Compounds with linking element: vowel (tweetotaller "someone who claims to abstain from tweeting", Tweetahead "a way to schedule tweets to be posted ahead of time with a simple Dashboard widget, for mac"); preposition (Tweeter-in-chief "a head of state who prefers to make official announcements to the nation via Twitter, because he thinks the media are biased against him"; drive-by-tweet "a fast tweet posted by an individual while he or she is in between tasks"); article (tweet-the-press "a method of sarcasm directed to the press media that delivers false news to unreliable news sources to see if they take the bait"); particle (Twitter-to-business "tweets to get more business leads by using Twitter")

- Particle compounds: tweetback "a list of links by other users to a particular tweet"; tweetup "an organized gathering of people that use Twitter"; tweetover "tweeting all night instead of sleeping"; tweet-out "a greeting to one's friends on the Twitter website".

\subsubsection{REDUPLICATIVE COMPOUNDS:}

- Reduplicative compounds proper: tweet tweet "another way of giving someone the word"; Twitter Twit "somebody who uses Twitter to keep everyone who follows them informed of their activity".

- Ablaut compounds: twittertweeter "somebody who posts too frequently on twitter"; Twitter-twatter "someone who Twitters so much that it makes them an obnoxious asshole twat"; Twitty-twat "a butchy, young-adult female that many people like to consider a heshe"; tweet twit "someone who acts like an idiot on Twitter by posting negative and idiotic comments"; tweet twat "a person who tweets way to much, usually about boring or everyday activities that no one cares about". 
- Rhyming compounds: speedtweet "a grouping of favorite or most contacted users"; Twitter-Bitter "being so sick of Twitter, even the mention of it brings on a nausea and sometimes a violent rage"; tweeter-totter "an argument on twitter between two people, usually about something funny or pointless"; Twitter Critter "person who spends too much time on Twitter, a cell-phone Twitter texting maniac, who believes they are the center of the universe"; Twitter twiker "somebody who repeatedly doesn't follow people back on Twitter when somebody follows them"; Twitty Litter "a tweet left in your name by a marketer"; tweet skeet "a spam account on Twitter"; tweet twitch "the process of reflexively posting and responding to Twitter tweets by pure spinal reflex without involvement of the brain".

- Rhyming combinations with linking conjunction: Tweet and Greet "a social media event where celebrities use Twitter to tweet their location so that fans can come and meet them"; tweet and meet "when you post your location on twitter, and someone at the same place tweets you wanting to meet up"; tweet and delete "the act of posting a tweet on Twitter and then deleting it".

The compounds form a large part of the new 'Twitter'-words I believe due to its simplicity and inherent obviousness during decoding that requires no extra-linguistic context. The leading role of compounding in the formation of the new 'Twitter' vocabulary can be associated with the rapid growth of complex concepts in various areas of public life over the recent decades. New 'Twitter' units formed from two or more stems convey the complex concepts in a concise form. This can be attributed mainly to the desire to economize language efforts, which is a universal phenomenon and can be traced in many languages, as well as to the semantic capacity of compounds. Their meaning is mostly motivated by their structure.

3.3. BLENDING. Blending is "on the verge of compounding and abbreviation and combines features of both these phenomena" (Bauer 1983:236). The semantic link of blends with the lexicalized model is evident from the relation among the semantically related components. In analyzed dataset we identified the following models of blends within two formal types:

\subsubsection{TOTAL BLENDS. In these both source words are reduced to splinters:}

- $a b+c d \rightarrow a c:$

Nouns: twapp = tw(itter) $+\operatorname{app}($ lication $)$ "an application that utilized Twitter"; twinfo = tw(itter) + info(rmation) "a small bit of information transmitted on Twitter".

- $\mathrm{ab}+\mathrm{cd} \rightarrow \mathrm{ad}$ :

Nouns: twam $=\mathrm{Tw}(\mathrm{itter})+(\mathrm{sp})$ am "Twitter spam"; twittionary $=$ Twitt(er) + (dic)tionary "a list of Twitter related words"; twaffic $=$ Twitter + traffic "Twitter traffic"; twemment = Twe(et) + (co)mment "Twitter comment"; tweetalog $=$ tweet + catalogue "Twitter lists, ie. card catalog for twitters/tweets"; twiatus $=\mathrm{Tw}$ (itter) + (h)iatus "a break from tweeting"; twoet/twoem/twoetry $=\mathrm{Tw}(\mathrm{itter})+$ (p)oet/(p)oem/(p)oet/ry "a poet, poem, or poetry on Twitter"; twewbie $=t w(i t t e r)+$ (n)ewbie "somebody new to Twitter"; Twellow=Tw(itter) + (f)ellow "a way to help find people with similar interests or business"; tweaming $=\mathrm{Tw}($ itter $)+($ dr)eaming "dreaming about Twitter"; adventuritter $=$ adventur(ous) + (tw)itter "an adventurous twitterer"; twidow (er) = twi(tter) + (wi)dow(er) "a Twitter widower"; twirgin = twi(tter) + (v)irgin "new to twitter"; twottie = Tw(itter) + (h)ottie "popular user that knows how to roll out the tweets"; dweet $=\mathrm{d}(\mathrm{rug})+(\mathrm{t})$ weet "a tweet made, perhaps regrettably, while under the influence of alcohol. 
Adjectives: twappy = Twitter + happy "being happy on Twitter"; tweelicious=tweet + delicious "feeling satisfaction by tweeting"; twocal $=\mathrm{Tw}(\mathrm{itter})+$ (1)ocal "describing local Twitter user"; twabulous = tweet + fabulous "fabulous tweet or information"; tweedundant $=$ Twee $(\mathrm{t})+(\mathrm{re})$ dundant "repeated tweet". Verbs: tweerun $=$ twee $(\mathrm{t})+(\mathrm{re})$ run "to rerun own tweets to illicit a greater response".

- $a b+c d \rightarrow a / d:$

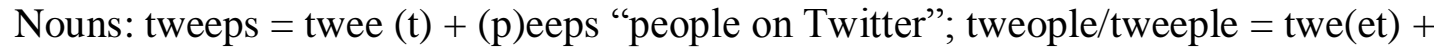
(p)eople "a group of people that are your Followers or you may be Following on Twitter"; twitchfork = twit(ter) + (p)itchfork "the use of Twitter as a tool for social protest"; twingo =twi(tter)+(1)ingo "twitter lingo".

Adjective: tweepish $=$ twee $(\mathrm{t})+(\mathrm{sh})$ eepish "feeling sheepish or regretful about something you tweeted".

Verbs: sleet $=$ slee $(p)+(t w) e e t$ "to twittering in your sleep, not remember doing it".

3.3.2 PARTIAL BLENDS. In these only one source word is reduced:

- $a b+c d \rightarrow$ acd:

Nouns: twapplications $=$ Tw(itter) + applications "Twitter applications";

twanxiety=Twitter+anxiety "excessive, exaggerated anxiety and worry about everyday Twitter life"; emergetweet = emerge(nce) + tweet "tweet sent out only in the case of an emergency when 911 is not available"; twinfancy = twi(tter) + infancy "tweeters who are in the early stages of learning how to twitter".

Adjectives: twaddicted = Tw(itter) + addicted "addicted to Twitter"; twaiting = tw(itter) + waiting "twittering while waiting".

Verbs: twadd = tw (itter) + add "to add/follow someone to your Twitter account as a friend"; detweet=delete+tweet "to delete a tweet".

- $a b+c d \rightarrow a / c d:$

Nouns: politweet $=$ polit(ical) + tweet "a political tweet"; twimmolation $=t w i(t t e r)+$ immolation "the act of damaging one's reputation through an offensive or otherwise inadvisable Twitter post"; twedit = twe $(\mathrm{t})$ + edit "making edits to a retweet to make it fit within the 140 character limit"; twake-up = tw(eet) + wake up "tweeting as soon as you wake up in the morning", itweet $=\mathrm{it}(\mathrm{ch})+$ tweet "an itch to tweet real-time embarrassing pictures, but doing so in public would look terribly awkward".

- $a b+c d \rightarrow a b c:$

Nouns: tweetrehab = twitter + rehab(ilitation) "a place you go to get treatment for tweeting"; twittercon = twitter + con(ference) "a conference of twitter users".

- $\mathrm{ab}+\mathrm{cd} \rightarrow \mathrm{abd}$ :

Nouns: twitterverse $=$ twitter $+($ uni) verse "the Twitter universe"; occasionitter $=$ occasion + (Tw)itter "an occasional tweeter"; twittertainment = twitter+(enter)tainment "entertainment provided by people on Twitter"; twittertude = twitter + (atti)tude "bad Twitter attitude or a state of being"; Twitterfly = Twitter + (butter)fly "being a social butterfly on Twitter evidenced by extreme usage of @ signs". Adjectives: Twittercized= Twitter + (criti)cized "criticized on Twitter for doing or saying something really dumb"; Twittermated =Twitter + (Auto)mated "automated Twitter responses that are out of context because a bot program is responding to the mention of their account and not that actual mention"; tweetcited = tweet $+($ ex $)$ cited "excited about Twitter". 
The rapid growth of new 'Twitter' blends in recent years can be attributed to the simplicity of the process which is the convenient means of conveying complex concepts in a concise form. However, it is not a mechanical process, but a rather creative one, reflecting a desire to provide emotional coloring, originality, humor. Thus, the formation of such units also proves the tendency to add emotion and diversity to the language, enriching its stylistic features.

3.4. ABBREVIATIONS. Abbreviations are defined as "lexical forms that are made up by fewer characters than the full form of a word or a combination of words" (Bieswanger, 2013). They are used on social media to "economize on typing effort" (Herring 2001: 617), to play with language, show creativity and indicate an intimate connection of in-group communication.

In analyzed dataset we divided abbreviations into the following types:

- Initialisms (often created by analogy with existing ones): RT "Retweet" > PRT "Partial retweet" or "please retweet" > "Real life retweet" > RTL "Retweet Love"; MT "Modified tweet" > MRT "modified retweet" $>$ TT "Translated tweet"; TFTT "Thanks For This Tweet" > TQRT "Thanks for the retweet"; TFF "Twitter Friends Forever" < slang BFF "Best Friends Forever"; TMB "Tweet me back" < TMB "text me back"; OCT "Obsessive Compulsive Twitterer $<$ OCD "Obsessive Compulsive Disorder"; P.T. "Post Tweet" $<$ P.S. "Post Scriptum that comes after your statement".

- Mixed formations: RTweepartee is "a term for carrying on an argument or discussion via Retweets (RT)"; TweetUI ('tweet you eye') is modelled after DUI for "a Tweet written and submitted under the influence." or "using Twitter with enough drinks, narcotics, stimulants, pain killers, mood enhancers to alter the normal 'Tweets"'; twit$A D$ "Twitter advertising network".

3.5. ANALOGY. Analogy is an effective morphological mechanism of 'Twitter' word-formation that is used when a new word is coined not on a particular model but is modelled on a precise actual word or phrase. The speakers often choose to create a new word which bears a resemblance to another particular item rather than using only word-formation rules. The analogy is used to select, to generate and to predict new forms on the basis of the similarity of a given base with existing forms in the lexicon. The new words that are rule-governed and motivated by analogy are the most probable and acceptable ones. In analogical word-formation the process of association of the new word (target) with the model one helps to understand newly coined words using the prior knowledge of model items (Mattiello 2018:11). Similarity features allow an association of the target word with the model word or pattern. We classified new Twitter words coined by analogy according to their morphological category and word-formation patterns:

\subsubsection{COMPOUNDING PATTERNS:}

- Nouns: twitterbox "a chatter box that uses twitter as their delivery mechanism" < chatter-box; tweetfart "state when you start typing a tweet and forget what you were typing" < brainfart; twittermob (usually derogatory) "a group of Twitter users targeting an issue or individual, especially one that forms suddenly and is viewed as hostile" $<$ flashmob; Tweetership "the followers you have on Twitter" < readership; tweeterview "an interview that takes place via Twitter" $<$ interview; tweetdroping "eavesdropping on someone else home page in friends mode" < eavesdropping; Tweeter-in-chief "a head of state who prefers to make official announcements to the nation via Twitter, because he thinks the media are biased against him" < Commander-in-chief; tweetup "an organized gathering of people that use Twitter" $<$ meet-up; tweetover "like a sleepover, except instead of chilling with a buddy you tweet all night" < sleepover; 
tweet-out "a greeting to one's friends on the Twitter website $<$ shout out; tweetback "a list of links by other users to a particular tweet" < trackback or linkback.

Some new 'Twitter' metaphors are coined by analogy with compounding patterns which exhibit a constant element such as a body part: tweethead "a word that can be to describe certain people on Twitter who tweet stupid, uninteresting" or "a person who is addicted to Twitter, and twittering" < pighead; tweethearts "celebrity Twitter supporters" < sweethearts; twitterbrain "a human being attached to mobile devices as if it were life support"; twitter mouth "someone who constantly has to tell everything that's going on in their life/what they're currently doing down to the second, never shut up" < sweet mouth.

- Adjective: tweet-happy is used to describe a person who just can't wait to post a new tweet and uses every spare moment for tweeting < trigger-happy.

- Verbs: to tweetspeak "to cram a "tweet" on Twitter in Twitter's 140 character limit" < to chat-speak; to twitterbomb "to pollute the stream of a Twitter hashtag by tweeting a meaningless, satirical, or spamlike tweet containing the hashtag" < to googlebomb; to drive-by-tweet "to post a fast tweet while being in between tasks" $<$ to drive-byhunting; to tweet back "to link to a particular tweet" < to linkback or trackback; to tweet out "to like on Twitter" < to sign out.

3.5.2 BlEnding PATTERnS. These include nouns: twitosphere "a global stock of Twitter users" $<$ blogosphere; twitterverse "the Twitter universe" < multiverse; Twittiquette "the manner in which you are supposed to communicate on Twitter" < netiquette; Twitizen "a Twitter user who not only uses Twitter to keep in touch with people they know in 'real' life but often to keep in touch with people they know through Twitter" < netizen; twittercide "deleting everything on Twitter page" < suicide; tweet-cred "attempt to impress a twitterer with more followers" < street-cred.

The target word can also be coined after a group of words that exhibit a phonestheme tw /tw/, i.e. a group of phonemes having recognizable semantic association with 'Twitter'/ 'tweet'. For example, the noun actwivist describes one who uses a Twitter message to advocate or oppose a political, social, or environmental cause (< activist); atwistocrat is a Twitter user who falsely sees self as superior and shows it with action or inaction ( $<$ twitter+ twisted +aristocrat).The adjective egotwistical (egoistical + twisted) describes one who has become so ultimately consumed by their ego, that their entire view of the world and everyone in it is twisted; intwituated means being in a state of infatuation with someone on Twitter; attwacted indicates sympathy (attraction) between two people on Twitter. The semantic relationship that link a target word with its corresponding model word may be motivated by semantic similarity (twittercide < suicide) or contradictory opposition (twittership < readership). Primary motivation of the new 'Twitter' words, which is pure similarity with a concrete model (precise lexical item 'Twitter' or 'twit' or 'tweet') and not with an abstract pattern, can be identified only in terms of substitution of part of a model word in the target word. The additional motivation is conformity to a rule patterns when the target word obeys word-formation rules.

4. Conclusion. This work concludes with the idea that in the era of ever-growing amount of data the popularity of Twitter has become a breeding ground for new brand-related words that have enriched the modern English vocabulary. The study of Twitter lexical innovations has determined productive patterns of new 'Twitter'/'tweet'-based word-formation such as affixation, compounding, blending, abbreviation and a powerful mechanism such as lexical 
analogy. We have also identified the most common models, on which the new units of each type are coined. These regular word-formation patterns of new 'Twitter' words are fully predictable. The irregular patterns are non-governed by the rule. They are creative and only partially predictable. These types of new 'Twitter' words are innovative, inventive and odd, especially for the comical (or even hilarious) effect that they are meant to produce. The coiners of new 'Twitter' words intend to be attractive, entertaining and effective at the same time, but also suggestive and evocative.

Blending and acronyms are particularly useful extra-grammatical operations that help not only to coin innovative and unusual 'Twitter' words, but also to create vocabulary that is cryptic, ambiguous, and enigmatic for those who are not familiar with it. Abbreviations are often vague and hard to interpret for outsiders. The use of acronymic units aids to establish an intimate and private connection, often without being too explicit or understood by non-members of the ingroup. Analogy as one of the main mechanisms used to create new eye-catching 'Twitter' words tend to establish closer social connection and help social closeness. The cases of the new 'Twitter' words involve reinterpretation of the model word before analogy applies or reanalysis of the target. The effect produced by ungrammatical morphological resegmentation is punning. The (pseudo)morphological similarity among the novel words provoke curiosity and amuse because of grammatical anomaly. When the new 'Twitter' word resembles its model word for some shared trait, it makes allusion to the model, or even want to satirize, parody its meaning or produce humor.

The results of the study show that original new 'Twitter'-words are coined to create verbal connection with other users, to establish social closeness and exclude those who are not part of the group ("Twitterverse"). The freshness of the new forms contributes to lowering the level of discourse to informal language and thus reinforces the users' relationship. Thus, creation of new 'Twitter' words attributes various functions and effects: desire to create or to express intimacy, group-restriction and privacy with fellow-users on Twitter; jocular/mocking function, which involves play on words (jocularity, playfulness and humor) or use of offensive expressions to scorn the group's outsiders (vulgarity); creativity, innovation and originality (freshness, novelty), desire to show off and boast, following fashion; denominative/labelling function ('twitter', 'twit', 'tweet', 'tw' elements show the connection with related brand name). The new 'Twitter'-words are created to find a new label which can express the new concept, but primary aim is less connected with denomination than with the users' amusement. The function of establishing a closer social connection appears to dominate. The effect that the new 'Twitter'-words more commonly produce is playfulness or fun, especially when the new targets function as echoes to model words that are implied or when their formation involves reanalysis. If target word and model word show both morphologic and phonological likeness it may facilitate the understanding of the new word. The knowledge of word-formation rules and patterns can also help the comprehension. Yet the language remains unique and inventive and new formations run the risk not becoming established new words if they are not reused or extended to a larger part of the speech community, although both forms are grammatical and completely transparent.

As to the factors that motivate the new 'Twitter'-words we identified the following:

- The phonological, morphological and semantic transparency of the new 'Twitter' terms in specialized IT language and amusing or a deliberately eye-/ear catching new 'Twitter' slang words in informal communication. 
- A strong tendency in the new 'Twitter'-words production to obey the regular patterns of word-formation rules, types, schemas. Irregular formations may be judged as conscious acts of creativity.

- New 'Twitter' forms are usually produced on the basis of productive patterns if there is no difficulty in segmenting and analyzing the product of word-formation type.

- For decoding of a new 'Twitter' word the sufficient contextual materials (definitions or explanations of the meaning, hyperlinks to definitions on the net) are provided.

\section{References}

Angelo, John. 1991. Fifty years among the new words: A dictionary of neologisms, 1941-1991. Cambridge: Cambridge University Press.

Bauer, Laurie. 1983. English word-formation. Cambridge: Cambridge University Press. Bauer, Laurie, Rochelle Lieber \& Ingo Plag. 2013. The Oxford reference guide to English morphology. Oxford: Oxford University Press.

Beal, Vangie. 2016. Twitter dictionary: A guide to understanding Twitter lingo. Webopedia. https://www.webopedia.com/quick_ref/Twitter_Dictionary_Guide.asp.

Bibliography of research on Twitter \& microblogging. http://www.danah.org/researchBibs/ twitter.php.

Bieswanger, Markus. 2013. Micro-linguistic structural features of computer-mediated communication. In Susan Herring, Dieter Stein \& Tuija Virtanen (eds.). Pragmatics of computer-mediated communication, 463-486. https://doi.org/10.1515/9783110214468.463.

Cannon, Garland. 1987. Historical changes and English word-formation: Recent vocabulary. New York: Lang.

Green, Jonathon. 2010. Green's dictionary of slang. Edinburgh: Chambers. https://beta.greensdictofslang.com/.

Grieve, Jack. 2016. Regional variation in written American English. http://www.cambridge.org/gb/academic/subjects/languages-linguistics/grammar-andsyntax/regional-variation-written-american-english.

Herring, Susan C. 2001. Computer-mediated discourse. Oxford: Blackwell.

Mattiello, Elisa. 2018. Analogy in word-formation. A study of English neologisms and occasionalisms. Berlin/Boston: Walter de Grueter GmbH.

McFedries, Paul. 2009. Twitter tips, tricks and tweets. http://mcfedries.com/cs/content/ TwitterTTT.aspx.

Oxford English dictionary online, $3^{\text {rd }}$ edn. Oxford: Oxford University Press http://www.oed.com/.

The Twitter dictionary aka Twittonary. http://twittonary.com/.

The Urban dictionary. https://www.urbandictionary.com.

Word spy: The word lover's guide to new words. https://www.wordspy.com/.

Zimmer, Ben. 2011.Twitterology: A new science? The New York Times.

https://www.nytimes.com/2011/10/30/opinion/sunday/twitterology-a-new-science.html. 